\title{
Physics under the bonnet of a stellar evolution code
}

\author{
Richard J. Stancliffe \\ Argelander-Institut für Astronomie, Auf dem Hügel 71, D-53121 Bonn, Germany \\ email: rjstancl@astro.uni-bonn.de
}

\begin{abstract}
Just how good are modern stellar models? Providing a rigorous assessment of the uncertainties is difficult because of the multiplicity of input physics. Some of the ingredients are reasonably well-known (like reaction rates and opacities). Others are not so good, with convection standing out as a particularly obvious example. In some cases, it is not clear what the ingredients should be: what role do atomic diffusion, rotation, magnetic fields, etc. play in stellar evolution? All this is then compounded by computational method. In converting all this physics into something we can implement in a 1D evolution code, we are forced to make choices about the way the equations are solved, how we will treat mixing at convective boundaries, etc. All of this can impact the models one finally generates. In this review, I will attempt to assess the uncertainties associated with the ingredients and methods used by stellar evolution modellers, and what their impacts may be on the science that we wish to do.
\end{abstract}

Keywords. stars: evolution, numerical methods

\section{The Basics}

At its most basic, the physics in a stellar evolution code is quite simple. The stellar structure is summed up by the four equations:

$$
\begin{aligned}
\frac{\mathrm{d} m}{\mathrm{~d} r} & =4 \pi \rho r^{2} \\
\frac{\mathrm{d} L}{\mathrm{~d} r} & =4 \pi \rho r^{2} \epsilon \\
\frac{\mathrm{d} P}{\mathrm{~d} r} & =-\frac{G m}{r^{2}} \rho \\
\frac{\mathrm{d} T}{\mathrm{~d} m} & =-\frac{T}{P} \frac{G m}{4 \pi r^{4}} \nabla
\end{aligned}
$$

which describe mass conservation, energy conservation, hydrostatic equilibrium and energy transport respectively. These equations alone say nothing about a star's evolution, which is governed by its change in composition. We therefore must have an equation to describe the change in mass fraction for each chemical species we wish to follow. In many codes, this is limited to a set of 7 energetically important species, including: ${ }^{1} \mathrm{H},{ }^{3} \mathrm{He} \dagger$, ${ }^{4} \mathrm{He},{ }^{12} \mathrm{C},{ }^{14} \mathrm{~N},{ }^{16} \mathrm{O}$ and ${ }^{20} \mathrm{Ne}$. Chemical species are affect by both nuclear reactions and mixing of material (primarily by convection), and this is commonly modelled using a

$\dagger{ }^{3} \mathrm{He}$ is not energetically important, but it can be used to drive a form of non-convective mixing in low-mass stars near the tip of the red giant branch, see e.g. Eggleton et al. (2006), Charbonnel \& Zahn (2007) and Stancliffe (2010). 
diffusion equation of the form:

$$
\frac{\partial}{\partial m}\left(\sigma \frac{\partial X_{i}}{\partial m}\right)=\frac{\mathrm{d} X_{i}}{\mathrm{~d} t}+R
$$

where $X_{i}$ is the mass fraction of species $i, R$ is the sum of all production and destruction of species $X_{i}$ via nuclear reactions, and $\sigma$ is the mixing coefficient.

In order to solve these equations in our codes, we first have to choose a numerical method by which to solve them. Meynet et al. (2004) have investigated three separate schemes and assessed their fitness for stellar physics problems. Most codes make use of an implicit finite difference scheme, with the star being divided up into several mesh points. For our diffusion equation, we obtain the following difference equation:

$$
\left(\frac{X_{k}-X_{k}^{0}}{\Delta t}+R_{X, k}\right) \delta m_{k}=\sigma_{k+\frac{1}{2}} \frac{X_{k+1}-X_{k}}{\delta m_{k+\frac{1}{2}}}-\sigma_{k-\frac{1}{2}} \frac{X_{k}-X_{k-1}}{\delta m_{k-\frac{1}{2}}}
$$

where $X_{k}$ and $X_{k}^{0}$ are the abundances at mesh point $k$ at the present and previous timesteps, $\Delta t$ is the timestep, $\delta m_{k \pm \frac{1}{2}}$ is the mass enclosed in the zones above and below mesh point $k$ and $\sigma_{k \pm \frac{1}{2}}$ are the diffusion coefficients associated with these zones.

Here we hit our first snag: we don't always have the information we want at the point that we want it. One can imagine we have divided our star up into a series of connected boxes. Each box contains material of a certain composition, and has certain physical conditions (specifically here a density and a temperature). The interfaces of these boxes are where we define the star's radius, mass co-ordinate and luminosity. Crucially here, our diffusion coefficient should describe the flow of matter through one of these interfaces, but it must be computed from information we don't have defined at the interface. One typically makes some kind of average between the quantities at adjacent mesh points. One can choose a geometric average, i.e. $\sigma_{k+\frac{1}{2}}=\sqrt{\sigma_{k} \sigma_{k+1}}$, or an arithmetic average, i.e. $\sigma_{k+\frac{1}{2}}=\frac{1}{2}\left(\sigma_{k}+\sigma_{k+1}\right)$, and neither choice is a priori better than the other. Yet the choice can have important consequences: for example, for thermally pulsing asymptotic giant branch stars, it can mean the difference between obtaining third dredge-up or not.

The next choice one has to make is whether one solves for all the equations of stellar structure in one go (the simultaneous approach), or whether one first computes a structure and then solves for the chemical evolution. In most cases, the two approaches will give the same results (Stancliffe 2006). However, there are cases, particularly when mixing and burning combine to give strong energetic feed back, when the simultaneous approach is to be preferred. One example of this is the occurrence of proton ingestion episodes in low metallicity AGB stars (e.g. Lau et al. 2009).

Having finally set up our equations and method of solution, the machinery of our stellar evolution code is almost complete. Two things remain to be determined, namely how we set the temporal and spatial resolution. The size of the timestep used is typically set with reference to the last converged model computed. If things are changing rapidly, one reduces the timestep size. Exactly which quantities one uses is a matter of choice. One can decided that if the sum of all changes to the previous model is larger than a certain value, the timestep should be reduced. In addition, requirements that certain properties of the model (such as the hydrogen- or helium-burning luminosity) should not change by more than a fixed amount in one timestep can also be used. Such selections can be very specific to an evolutionary phase, for example, rapid variations in helium-burning occur during core-helium flashes and during thermal pulses on the asymptotic giant branch.

In codes that use a Lagrangian scheme, determining the spatial resolution is relatively straightforward. If a given physical quantity (e.g. the hydrogen abundance) varies by 
more than a predetermined amount between two mesh points, one can simply introduce an additional mesh point. The downside of this method is that one may have to introduce a large number of mesh points to adequately resolve certain features. More mesh points means more computational time is required. A rather unique method for determining the placing of mesh points was developed by Eggleton (1971). In his code, a fixed number of mesh points are used and their location is moved around to follow features of interest. This is achieved by the use of a mesh spacing function Q. Points are placed such that the gradient $\mathrm{d} Q / \mathrm{d} k$ is the same from mesh point to mesh point. Thus, more points are placed in regions where $\mathrm{Q}$ changes rapidly. With such a method it is possible to compute the evolution of an asymptotic giant branch star with just 1000 mesh points (Stancliffe et al. 2004; Stancliffe \& Jeffery 2007).

It might seem obvious that getting the spatial and temporal resolution correct is important, but it is something that is often overlooked. Convergence tests are often not performed and this can lead to errors creeping in to published results. A recent example of the effects of poor spatial and temporal resolution was provided by Lattanzio et al. (2015). They showed that for extra mixing at the tip of the giant branch, the predicted behaviour for the lithium abundance was dependent on both spatial and temporal resolution. This was shown to be true for a number of different evolution codes. The moral of this story is clear: always test results for convergence!

\subsection{Convection and convective overshooting}

Convection is almost certainly the great Achilles' Heel of stellar evolution calculations, and unfortunately it is likely to remain so for some time. The majority of stellar codes still rely on the venerable mixing length theory of Böhm-Vitense (1958). Mixing length theory (MLT) is an excellent prescription for implementation in stellar modelling. It is a local theory, that is to say, it allows one to compute the temperature gradient at any given mesh point without having to refer to conditions at any other mesh point. This presents a considerable saving in computational cost. All one has to do is to calibrate the mixing length parameter (but be warned! there are still further parameters in the theory, see Salaris \& Cassisi 2008, for details), using the Sun as a reference, and your stellar evolution code is ready to model whatever phase of evolution you are interested in.

It should be noted that one of the assumptions of MLT theory is that the mixing length obtained by calibration to the Sun is applicable to all convective situations, regardless of stellar mass, metallicity or evolutionary state. There is some observational evidence to suggest that this may not be the case (e.g. McSaveney et al. 2007, require high mixing lengths to reproduce the colours of their asymptotic giant branch stars). In addition, radiation hydrodynamics simulations of convective envelopes also suggest that the mixing length should be a function of $\mathrm{T}_{\text {eff }}, \log \mathrm{g}$ and $[\mathrm{Fe} / \mathrm{H}]$ (see Salaris \& Cassisi 2015, for a discussion of the various simulations and their results).

Among the alternative theories of convection is the so-called full spectrum of turbulence (FST) model of Canuto \& Mazzitelli (1991). While MLT assumes (essentially) only one large eddy, the FST model includes the contributions of turbulent eddies of all sizes. At high convective efficiencies, the FST model may produce convective fluxes that are 10 time larger than predicted by MLT (Canuto \& Mazzitelli 1991). By fixing the mixing length of the FST model to be the distance to the top of the convection zone, Canuto \& Mazzitelli (1991) found they were able to produce a viable Solar model without recourse to free parameters. More recently, Pasetto et al. (2014) have also produced a parameterfree theory of convection, but it has yet to be tested in full stellar evolution models. Most recently, Arnett et al. (2015) have produced a 1D convective theory based on 
3D hydrodynamical simulations of convection. The implementation of such convective theories and their effect on stellar models remains to be determined.

Overshooting is perhaps the most commonly included non-convective (or extra) mixing mechanism included in stellar codes. Unfortunately, the term is often misused or at least misunderstood. In many cases, the use of overshooting is not necessarily related to convection and is merely a means of extending the size of a chemically homogenous region. Such an extension may be caused by a variety of processes, the chief example of which would be rotational mixing. However, there are a variety of forms of overshooting that are indeed directly linked to convection. Viallet et al. (2015) provide an excellent discussion of the three cases of true convective overshooting.

As with convection, the introduction of an overshooting prescription to a code brings with it the introduction of a free parameter (hopefully no more than one!). One must then find some way of calibrating this parameter. There are various ways to do this, including the use of colour-magnitude diagrams for stellar clusters (e.g. VandenBerg et al. 2006), or via the use of eclipsing binary systems (e.g. Schröder et al. 1997; Claret 2007). Such calibrations are not straightforward, as one often has imperfect knowledge of the composition of the target objects. One is therefore left to run a grid of models with a range of overshooting parameters and metallicities, and to determine the best fitting model from this grid. Recently, Stancliffe et al. (2015) performed such a calibration using a sample of eclipsing binaries with well-determined parameters from the sample of Torres et al. (2014), using two separate stellar evolution codes with different prescriptions for the overshooting.

Seismology may offer a better way to improve calibration of overshooting (or other interior mixing physics). A recent example of this is the work of Moravveji et al. (2015). These authors were able to use the period spacing observed in the slowly pulsating B star KIC 10526294 to put constraints on interior mixing. Their seismic modelling was able to determine the free parameter, $f$, for the diffusive prescription of overshooting (see e.g. Herwig 2000, for a description of this prescription). Moreover, they find that the diffusive prescription better represents the data that a simple step-function overshooting model.

As with convection itself, one typically calibrates the overshooting on a particular evolutionary phase and then continues to use that prescription for other phases. Should the extent of overshooting be the same for a helium-burning core as it is for a hydrogen burning core? Again, seismology may be able to help stellar modellers. Thanks to seismology, it is possible to distinguish between core-helium burning red giants, and those on the first ascent of the giant branch (Bedding et al. 2011). Constantino et al. (2015) looked at predictions for four different treatments of core overshooting and their predictions of the asymptotic g-mode $l=1$ period spacing. They concluded that convective cores may be more massive than stellar models currently predict. Clearly, asteroseismology has a major role to play in helping stellar evolution modellers deal with problematic phases of evolution.

\subsection{Microphysics inputs}

The final component for stellar codes that needs to be discussed is the microphysics input. By this, I mean the nuclear reaction rates, opacities and the equation of state (EoS). These contributions supply the missing quantities required for our stellar equations. Of these three things, the reaction rates are probably of the least concern for stellar modelling. As mentioned above, the reactions of only a handful of species contribute enough energy to significantly affect the structure of a star. The majority of these are well studied, and known to a good degree of accuracy. Nuclear reaction rates are more of a problem for 
those interested in stellar nucleosynthesis, where the range of isotopes to be included is much greater.

Opacities are perhaps one of the greater areas for concern among the microphysics inputs for the simple reason that there are not many sources that provide the necessary data over the ranges and temperatures of interest (to say nothing of composition!). There are really only two sets of opacity tables currently available to stellar modellers: the OPAL tables of Iglesias \& Rogers (1996), and the OP tables of Badnell et al. (2005). For a discussion of the impacts of opacities on asteroseismology, the reader is directed to W. Ball's contribution in these proceedings.

Among the possible choices for the equation of state, the OPAL EoS (Rogers et al. 1996) is the most commonly used. Other choices include modified versions of the Eggleton et al. (1973) EoS and the FreeEoS†. Helioseismology strongly favours the OPAL EoS (see S. Basu's contriubtion to these proceedings). For more advanced evolutionary phases, the EoS is not thought to be a major contributor to the uncertainty though this certainly warrants further study.

\subsection{Additional physics}

I have purposefully kept the discussion above to the absolute basics of a stellar code. Many stellar codes go far beyond this in their list of included physics. Additional physics that is now commonly included in stellar codes includes: gravitational settling, rotation and binary physics. Magnetic fields are also increasingly being included in certain codes. A discussion of the details of each of these phenomenon would take far longer than the number of pages allotted to this contribution.

\section{Stellar models in practice}

So what happens when we put all of the above together? Everything sounds straightforward, so we might expect that stellar codes should produce extremely similar results. In Fig. 1 I show evolutionary tracks for a $1 \mathrm{M}_{\odot}$ star from a collection of popular evolution codes. With the exception of the tracks computed with STARs (Eggleton 1971; Stancliffe \& Eldridge 2009) and MESA (Paxton et al. 2011), these all come from publicly available databases. It is assumed that the $1 \mathrm{M}_{\odot}$ model from any given grid is an accurately calibrated Solar model, though it is often not clear if this is the case. For the BASTI model, the provided track is not the one used for the Solar calibration, as it does not include the effects of gravitational settling though these were used for the calibration (see Pietrinferni et al. 2004, for details). To reproduce the spread in the stellar tracks with a single code (in this case the STARS code) would require models with initial masses of $0.97-1.02 \mathrm{M}_{\odot}$.

So what is the origin of the spread? One problem is the choice of which parameters to use for the solar calibration. Minimally, one needs to fit the Solar luminosity, effective temperature (the solar radius can be substituted for one of these two) and the surface $Z / X$. However, one can also include details derived from helioseismology, such as the depth of the Solar convection zone, the helium content of the convection zone and even the Solar sound speed profile. In the left-hand panel of Fig. 2, I show stellar tracks computed using MESA (J.-C. Passy, private communication) for a variety of calibration constraints and for the Solar abundances determined by Asplund et al. (2009). While the fit including the Solar sound speed seems reasonable when one looks at the track in the

$\dagger$ The FreeEoS fortran libraries are available from http://freeeos.sourceforge.net/ 


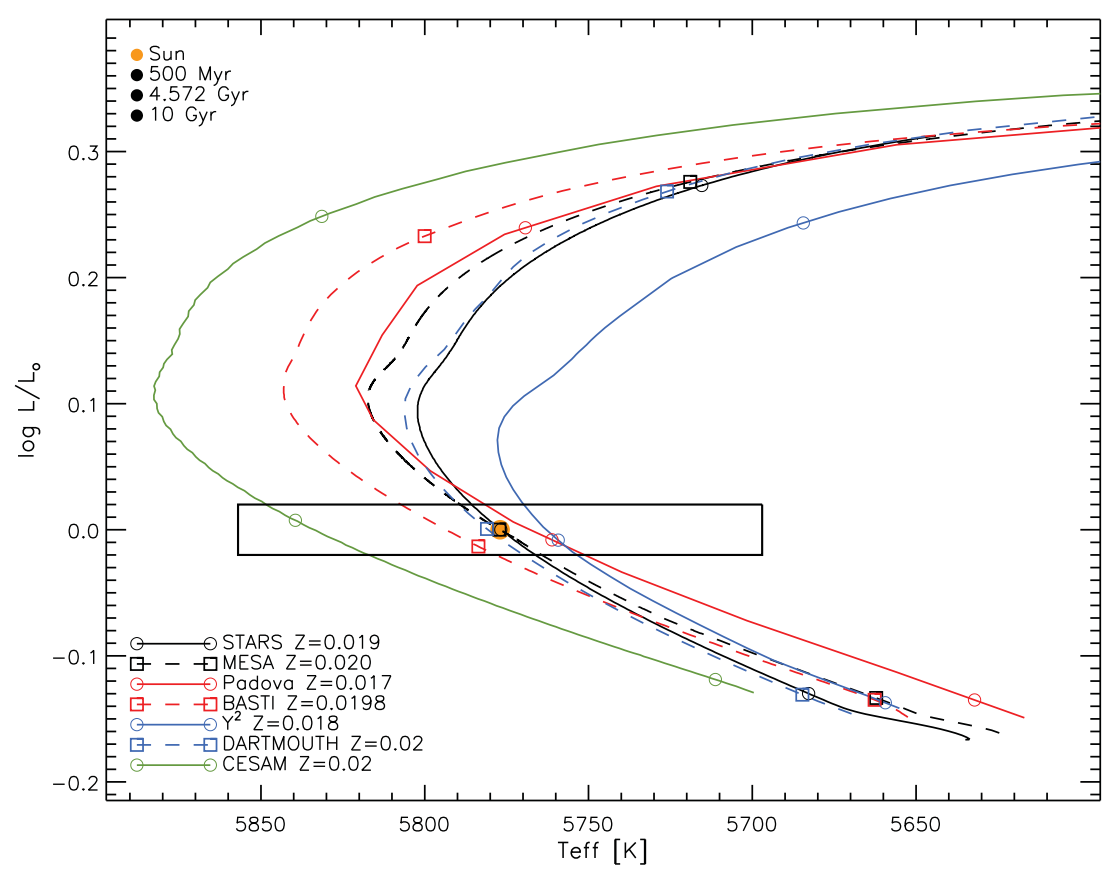

Figure 1. Evolutionary tracks from a collection of popular stellar codes. On each track, symbols mark three key ages: $500 \mathrm{Myr}, 4.572 \mathrm{Gyr}$ and $10 \mathrm{Gyr}$. The box represents an expected error bar for the GAIA mission, centred on the Sun.
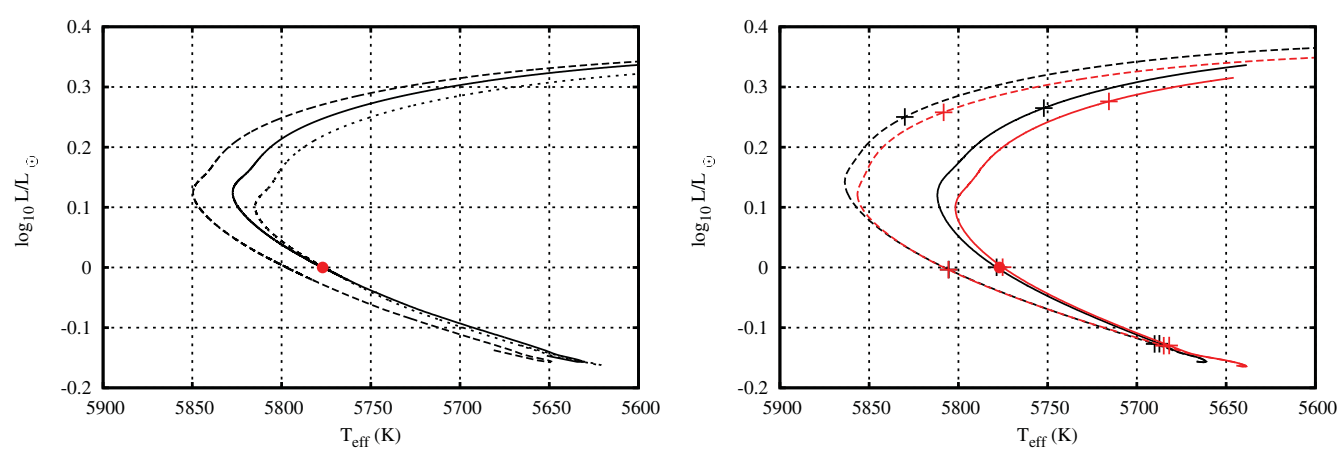

Figure 2. Left: Solar tracks computed using MESA with various different calibration constraints. The solid line includes only $L_{\odot}, \mathrm{T}_{\text {eff }}$ and $Z / X$, while the dashed line also adds the depth of the convection zone, and the dotted line includes the Solar sound speed profile. Right: Solar tracks computed with and without gravitational settling (solid and dashed lines respectively), but assuming a mixing length calibrated including gravitational settling. Red indicates A09 abundances, while black is for GN93 abundances. In both figures the red dot indicates the position of the Sun.

HR diagram, it's surface $Z / X$ is around 0.025 . This value is clearly incompatible with the Asplund et al. (2009) value of 0.0181, and so this is not a good Solar model.

Another source of error is introduced if the physics included in the calibration of the mixing length parameter is not the same as the physics used for subsequent computations. The right-hand panel of Fig. 2 shows an example of this. In this case, the calibration was performed using gravitational settling (solid lines) and then re-run without settling 


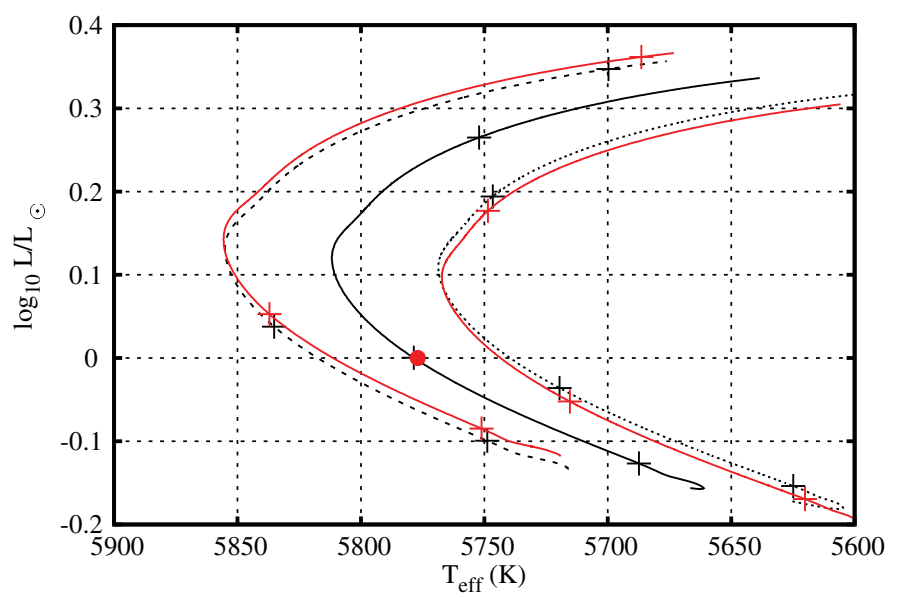

Figure 3. Evolutionary tracks for a $1 \mathrm{M}_{\odot}$ model showing the effect of a small change in the hydrogen abundance. The solid line indicates the calibrated model, while the dotted and dashed lines show models where the initial hydrogen abundances has been increased and decreased by 0.01 (a change of $14 \%$ from the calibrated value) respectively. Pluses mark ages of $500 \mathrm{Myr}$, 4.57 Gyr and 10 Gyr. The red circle represents the location of the Sun. Red lines mark stellar tracks with masses of $0.98 \mathrm{M}_{\odot}$ and $1.02 \mathrm{M}_{\odot}$.

included (dashed lines). A shift of around $25 \mathrm{~K}$ is obtained at the Solar age, and the discrepancy grows to around $50 \mathrm{~K}$ by the time the track reaches its hottest point.

One final thing to consider when trying to fit a stellar model to an observed object is the ignorance of the object's chemistry. Fig 3 shows the effect of a $14 \%$ shift in the initial hydrogen abundance (this is a change of 0.01 in the initial hydrogen mass fraction) on a $1 \mathrm{M}_{\odot}$ model of Solar metallicity. The tracks with the shifted hydrogen abundance can almost be reproduced by tracks of 0.98 and $1.02 \mathrm{M}_{\odot}$. This means that an ignorance of a star's initial hydrogen abundance can translated into an error of $2 \%$ in determining the star's mass from the HR diagram. A similar result is obtained when one changes the initial metallicity. This may seriously inhibit our ability to accurately model stars: rarely do we have a complete knowledge of a star's composition.

\section{Conclusions}

I have given a brief review of the internal workings of a stellar structure code, highlighting some of the choices that have to be made before one can start to model a star. I have described the input physics one uses and highlight areas where improvements can be made. Asteroseismology will almost certainly play a major role in helping us make these improvements, as no other technique is capable of giving us such a direct window into a star's interior. Even with the basic physics currently included in current codes, there is a large degree of variation in the predictions for a simple $1 \mathrm{M}_{\odot}$ track. Some of this variation can be attributed to the choice of included physics, while some relates to just how the calibration of mixing length theory is performed. Even if these issues can be ironed out, one is still left with uncertainties relating to the composition of the stars one wishes to study.

\section{References}

Arnett, W. D., Meakin, C., Viallet, M., et al. 2015, ApJ, in press, arXiv:1503.00342 
Asplund, M., Grevesse, N., Sauval, A. J., \& Scott, P. 2009, Annual Reviews of Astronomy \& Astrophysics, 47, 481

Badnell, N. R., Bautista, M. A., Butler, K., et al. 2005, MNRAS, 360, 458

Bedding, T. R., Mosser, B., Huber, D., et al. 2011, Nature, 471, 608

Böhm-Vitense, E. 1958, Zeitschrift für Astrophysik, 46, 108

Canuto, V. M. \& Mazzitelli, I. 1991, ApJ, 370, 295

Charbonnel, C. \& Zahn, J. 2007, A\&A, 467, L15

Claret, A. 2007, A\&A, 475, 1019

Constantino, T., Campbell, S. W., Christensen-Dalsgaard, J., Lattanzio, J. C., \& Stello, D. 2015, MNRAS, 452, 123

Eggleton, P. P. 1971, MNRAS, 151, 351

Eggleton, P. P., Dearborn, D. S. P., \& Lattanzio, J. C. 2006, Science, 314, 1580

Eggleton, P. P., Faulkner, J., \& Flannery, B. P. 1973, A\& A, 23, 325

Herwig, F. 2000, A\&A A, 360, 952

Iglesias, C. A. \& Rogers, F. J. 1996, ApJ, 464, 943

Lattanzio, J. C., Siess, L., Church, R. P., et al. 2015, MNRAS, 446, 2673

Lau, H. H. B., Stancliffe, R. J., \& Tout, C. A. 2009, MNRAS, 396, 1046

McSaveney, J. A., Wood, P. R., Scholz, M., Lattanzio, J. C., \& Hinkle, K. H. 2007, MNRAS, 378,1089

Meynet, G., Maeder, A., \& Mowlavi, N. 2004, A $\& A, 416,1023$

Moravveji, E., Aerts, C., Pápics, P. I., Triana, S. A., \& Vandoren, B. 2015, A\& A, 580, A27

Pasetto, S., Chiosi, C., Cropper, M., \& Grebel, E. K. 2014, MNRAS, 445, 3592

Paxton, B., Bildsten, L., Dotter, A., et al. 2011, ApJS, 192, 3

Pietrinferni, A., Cassisi, S., Salaris, M., \& Castelli, F. 2004, ApJ, 612, 168

Rogers, F. J., Swenson, F. J., \& Iglesias, C. A. 1996, ApJ, 456, 902

Salaris, M. \& Cassisi, S. 2008, A\& $A$, 487, 1075

Salaris, M. \& Cassisi, S. 2015, A\&BA, 577, A60

Schröder, K.-P., Pols, O. R., \& Eggleton, P. P. 1997, MNRAS, 285, 696

Stancliffe, R. J. 2006, MNRAS, 370, 1817

Stancliffe, R. J. 2010, MNRAS, 403, 505

Stancliffe, R. J. \& Eldridge, J. J. 2009, MNRAS, 396, 1699

Stancliffe, R. J., Fossati, L., Passy, J.-C., \& Schneider, F. R. N. 2015, A\& A, 575, A117

Stancliffe, R. J. \& Jeffery, C. S. 2007, MNRAS, 375, 1280

Stancliffe, R. J., Tout, C. A., \& Pols, O. R. 2004, MNRAS, 352, 984

Torres, G., Vaz, L. P. R., Sandberg Lacy, C. H., \& Claret, A. 2014, AJ, 147, 36

VandenBerg, D. A., Bergbusch, P. A., \& Dowler, P. D. 2006, ApJS, 162, 375

Viallet, M., Meakin, C., Prat, V., \& Arnett, D. 2015, A\& A, in press, ArXiv:1506.03100 